\title{
openheart Characteristics and in-hospital outcomes of patients undergoing balloon pulmonary angioplasty for chronic thromboembolic pulmonary hypertension: a time-trend analysis from the Japanese nationwide registry
}

Shun Minatsuki (D) , Mitsuyoshi Takahara, ${ }^{2}$ Arihiro Kiyosue, ${ }^{1}$ Satoshi Kodera, ${ }^{1}$
Masaru Hatano, ${ }^{3}$ Jiro Ando, ${ }^{1}$ Shun Kohsaka, ${ }^{4}$ Hideki Ishii ${ }^{5},{ }^{5}$ Toshiro Shinke,
Tetsuya Amano, ${ }^{7}$ Yuji Ikari, ${ }^{8}$ Issei Komuro, ${ }^{1}$ J-SHD and J-PCl Investigators

To cite: Minatsuki S, Takahara M, Kiyosue A, et al. Characteristics and in-hospital outcomes of patients undergoing balloon pulmonary angioplasty for chronic thromboembolic pulmonary hypertension: a time-trend analysis from the Japanese nationwide registry. Open Heart 2021;8:e001721. doi:10.1136/ openhrt-2021-001721

Received 14 May 2021 Accepted 27 August 2021

Check for updates

(C) Author(s) (or their employer(s)) 2021. Re-use permitted under CC BY-NC. No commercial re-use. See rights and permissions. Published by BMJ.

For numbered affiliations see end of article.

Correspondence to Dr Shun Kohsaka; sk@keio.jp

\section{ABSTRACT}

Background Balloon pulmonary angioplasty (BPA), a novel technique initially introduced as a treatment for inoperable chronic thromboembolic pulmonary hypertension, is now increasingly being performed in a broader spectrum of patients. Here, we performed a time-trend analysis of the characteristics and in-hospital outcomes of patients who underwent BPA in Japan, using data extracted from nationwide procedure-based registration system.

Methods The Japanese Structural Heart Disease (JSHD) registry was established and sponsored by the Therapeutics and aims to provide basic statistics on the performance of structural interventions in Japan. J-SHD registers cases from approximately 200 institutions, representing more than $90 \%$ of SHD interventionperforming hospitals in the nation. We analysed the registered BPA data elements from January 2015 to December 2018. Successful BPA was defined as a session in which a physician successfully treated all targeted lesions.

Results There were a total of 2512 BPA sessions; the number of institutions and registered sessions increased from 30 to 50 sites and from 479 to 852 sessions during the study period, respectively. The average age of the patients was $66 \pm 13$ years, and $72.1 \%$ were women. In-hospital death was observed in $0.2 \%$, and the total complications rate was $5.3 \%$. The preoperative and postoperative mean pulmonary artery pressure were $32 \pm 11 \mathrm{~mm} \mathrm{Hg}$ and $30 \pm 10 \mathrm{~mm} \mathrm{Hg}$, respectively. Conclusion The number of BPA sessions increased during the study period, with an acceptable in-hospital complication rate.

\section{INTRODUCTION}

Since its approval in April 2010, balloon pulmonary angioplasty (BPA) for chronic Japanese Association of Cardiovascular Intervention and

\section{Key questions}

What is already known about this subject?

- The range of indications for balloon pulmonary angioplasty (BPA) in the treatment of chronic thromboembolic pulmonary hypertension (CTEPH) has been increasing, along with a development in the procedure technique. Japanese Structural Heart Disease registry provides statistics on the performance of structural interventions.

What does this study add?

- The number of BPA sessions increased with an acceptable complication rate.

How might this impact on clinical practice?

- BPA is a feasible and safe therapeutic method for treating patients with CTEPH.

thromboembolic pulmonary hypertension (CTEPH) has been performed at numerous operators and facilities in Japan ${ }^{1}$ and has become a valid therapeutic option for the management of CTEPH. Previous reports have demonstrated the effectiveness of BPA in improving the patients' haemodynamic and functional status, ${ }^{2-6}$ which in turn are associated with an improvement in the quality of life and prognosis of patients with CTEPH. ${ }^{7} 8$ Incorporating favourable outcomes from these reports, the European Society of Cardiology and the European Respiratory Society 2016 guidelines for the diagnosis and treatment of pulmonary hypertension state that BPA may be accepted as a therapeutic strategy. ${ }^{9}$

Given the technical advancement and better triaging of patients, BPA has become 
more widely recognised and is being performed in an increasing number of patients. Contemporary data provide compelling evidence on the benefits of BPA, with improvements in both symptoms and haemodynamic parameters. ${ }^{10} 11$ Accordingly, the range of cases in which BPA is indicated has been expanding; this range is reported to vary from $10 \%$ to $50 \% .^{12} 13$

The selected treatment policy for CTEPH differs in countries or facilities due to variations in the population of patients deemed inoperable for CTEPH in whom BPA can be performed. For example, pulmonary endarterectomy, the choice of treatment for CTEPH, is predominantly performed in Europe and the USA, ${ }^{1214}$ whereas in Japan, pulmonary endarterectomy is performed in only about $50-60$ cases per year. ${ }^{15}$

Given the increasing application of BPA among a broader spectrum of patients with CTEPH, it is necessary to track its role in real time because it has changed over time since the establishment of BPA procedures. Thus, this study aimed to investigate the temporal trends in characteristics and in-hospital outcomes of patients with CTEPH who underwent BPA.

The primary aim of this study was to describe the patient and procedural characteristics of BPA performed in Japan using the mandated nationwide registration system. The secondary aims were to establish BPA as a safe treatment for all Japanese patients with CTEPH, to collect reliable data and accurately describe the entirety of BPAs performed within country, and to seek for unmet needs regarding BPA and further conduct in-depth analysis to find potential solutions.

\section{METHODS}

\section{Data collection methods}

We collected information on the background of the target patients and the procedural results of BPA from 2015 to 2018. The data elements were collected from the Japanese Structural Heart Disease (J-SHD) registry, which was established in 2012 and is a prospective Japanese nationwide multicentre registry founded by the Japanese Association of Cardiovascular Intervention and Therapeutics (CVIT) designed to collect clinical variables and shortterm outcome data on patients with SHD. The mission of CVIT is to further the understanding of application of cardiovascular intervention and to establish safety of the procedure. Today, the database has grown to become one of the largest healthcare procedural databases with more than 20000 SHD intervention cases registered annually (excluding transcatheter aortic valve replacement, which is registered on J-TVT registry) from approximately 200 institutions in Japan representing more than $90 \%$ of all SHD intervention-performing hospitals in the nation. Importantly, case registrations in the J-SHD registry are essential for coronary interventionalist and educating hospital certification.

This study protocol was approved by the institutional review board (http://www.cvit.jp/files/registry/central_ irb.pdf). The BPA data, which are one of the components of the J-SHD, were collected from 2015. The CVIT registry subcommittee designed a software for a web-based data collection system through the National Clinical Database. Data managers in the participating hospitals submit data through this system annually. Because registration in the J-SHD database is mandatory for board certification and renewal applications, data completeness is high, although participation in the J-SHD is voluntary. The data analysis was performed in accordance with the Declaration of Helsinki and was approved by the ethics committee of the Clinical Research Promotion Network Japan (approval number CRPNJ_SOP-4).

\section{Study population}

We analysed the data of BPA sessions for patients with CTEPH registered in the J-SHD between January 2015 and December 2018. As there were no exclusion criteria, all sessions performed during this time interval were included in the present analysis (2512 sessions).

\section{Definition of variables}

Smoking was defined as any history of smoking. Chronic obstructive pulmonary disease was determined when the percentage of forced expiratory volume in $1 \mathrm{~s}$ was below $70 \%$ and other diseases that induce chronic airflow obstruction were excluded. ${ }^{16}$ Hypertension was determined when the systolic blood pressure was $\geq 140 \mathrm{~mm}$ $\mathrm{Hg}$, diastolic blood pressure was $\geq 90 \mathrm{~mm} \mathrm{Hg}$ or patients were treated with antihypertensive medications. ${ }^{17}$ Diabetes mellitus was determined when the fasting blood glucose level was $\geq 126 \mathrm{mg} / \mathrm{dL}$, non-fasting glucose level was $\geq 200 \mathrm{mg} / \mathrm{dL}$, haemoglobin A1c level was $\geq 6.5 \%$, glucose level 2 hours after a $75 \mathrm{~g}$ oral glucose tolerance test was $\geq 200 \mathrm{mg} / \mathrm{dL}$ or patients were treated with antidiabetic medications. ${ }^{18}$ Dyslipidaemia was determined when the fasting triglyceride level was $\geq 150 \mathrm{mg} / \mathrm{dL}$, fasting highdensity lipoprotein (HDL) cholesterol level was $<40 \mathrm{mg}$ / $\mathrm{dL}$, fasting low-density lipoprotein cholesterol level calculated from the Friedewald equation was $\geq 140 \mathrm{mg} / \mathrm{dL}$, non-HDL cholesterol level was $\geq 170 \mathrm{mg} / \mathrm{dL}$, or patients were treated with antihyperlipidaemic medications. ${ }^{19}$ Definitions of hypertension, diabetes mellitus and dyslipidaemia were the same as those in the Japanese Percutaneous Coronary Intervention registry. ${ }^{20}$ Maintenance dialysis included both haemodialysis and peritoneal dialysis. Coronary artery disease was defined as a history of myocardial infarction or revascularisation for stenotic coronary artery lesions, whereas cerebrovascular disease was defined as a history of cerebral infarction or haemorrhage with neurological symptoms sustained for $\geq 24$ hours. Data on the procedural time were collected after 2017, and data on cardiogenic shock (requiring mechanical support) were collected after 2018.

\section{Clinical outcomes}

Emergent BPA was defined as any BPA session that was not scheduled on the previous day. Successful BPA was 
defined as a BPA session in which a physician successfully treated all targeted lesions. In-hospital death included deaths caused by complications related to BPA or any comorbidity. Emergent surgical treatment was defined as any unscheduled surgery for BPA-related complications. Distal embolism was defined as an angiographically confirmed thrombotic event occurring in the pulmonary artery during or immediately after the session; acute obstruction was defined as thrombosis occurring during the perioperative phase (typically within 24-48hours). Vessel rupture was defined as dissection of the pulmonary artery induced by contrast medium injection and devices such as a guiding catheter, guiding wire and balloon. Contrast-induced nephropathy was defined as an elevation of serum creatinine of more than $25 \%$ or $\geq 0.5 \mathrm{mg} /$ $\mathrm{dL}(44 \mathrm{~mol} / \mathrm{L})$ from baseline within 48 hours. ${ }^{21}$ If the registrants selected 'Others' in the report form, there was a space allowing registrants to freely respond. Lung injury, which is a well-known BPA-specific complication manifested by bloody sputum, was not covered in the predefined procedural complication variables; it was recorded by text entry after selecting the 'Others' option in the complications recording.

\section{Statistical analysis}

We aimed to perform a time-trend analysis of BPA for CTEPH in Japan for the number of BPA sessions, performing institutions, patient characteristics (prevalence of background condition), procedural results and complication rates. Analysis of the relationship between the number of complications and the number of BPA sessions in each institution was also performed.

We divided the institutions into two groups: highvolume institutions that performed more than $50 \mathrm{BPA}$ sessions and general institutions that performed less than 50 sessions per year. The definition of high-volume centres as those that performed more than 50 BPA sessions was based on the facility certification standard of the Japanese Circulation Society (http://www.j-circ. or.jp/BPA/files/BPA_shidousisetsu_jissii_sidoui.pdf). The incidence of complications was compared between the two groups. Data are presented as mean $\pm \mathrm{SD}$ and medians (interquartile ranges) for continuous variables and as frequencies (proportions) for categorical variables. A value of $p<0.05$ was considered statistically significant.

Regarding time-trend analysis, $\mathrm{p}$ values for trend were derived from linear regression models for continuous variables and logistic regression models for categorical variables. The $95 \%$ CIs of the proportions of procedural success and complications were calculated using the Clopper-Pearson's exact method. The comparison between the groups was performed using an unpaired $t$ test. All statistical analyses were performed using R V.3.6.0 (R Development Core Team, Vienna, Austria).

\section{RESULTS}

\section{Baseline characteristics}

The baseline characteristics of patients are shown in table 1 .

The number of participating institutions and registered sessions increased every year (2015: 479 sessions, 30 institutions; 2016: 467 sessions, 42 institutions; 2017: 714 sessions, 48 institutions; 2018: 852 sessions, 50 institutions; figure 1A). The average age and percentage of female patients were not significantly different between years (2015: 66 \pm 12 years, 72.2\%; 2016: 64 \pm 14 years, $68.7 \%$; 2017: $66 \pm 13$ years, $74.5 \%$; 2018: $66 \pm 14$ years, $71.7 \% ; \mathrm{p}=0.66)$. The trends of the above results are shown in figure 1B,C.

\begin{tabular}{|c|c|c|c|c|c|}
\hline Year & 2015 & 2016 & 2017 & 2018 & P for trend \\
\hline Number of institutions & 30 & 42 & 48 & 50 & \\
\hline Sessions & 479 & 467 & 714 & 852 & \\
\hline Female (\%) & $346(72.2)$ & $321(68.7)$ & $532(74.5)$ & $611(71.7)$ & 0.66 \\
\hline Age (years) & $66 \pm 12$ & $64 \pm 14$ & $66 \pm 13$ & $66 \pm 14$ & 0.098 \\
\hline Median & $68(57-75)$ & $67(55-76)$ & $69(56-76)$ & $70(57-77)$ & \\
\hline Smoking (\%) & $31(6.5)$ & $60(12.8)$ & $78(10.9)$ & $111(13.0)$ & 0.002 \\
\hline COPD (\%) & $20(4.2)$ & $25(5.4)$ & $20(2.8)$ & $46(5.4)$ & 0.58 \\
\hline Hypertension (\%) & $103(21.5)$ & $153(32.8)$ & $217(30.4)$ & $298(35.0)$ & $<0.001$ \\
\hline Diabetes mellitus (\%) & $22(4.6)$ & $45(9.6)$ & $70(9.8)$ & $112(13.1)$ & $<0.001$ \\
\hline Dyslipidaemia (\%) & $74(15.4)$ & $116(24.8)$ & $167(23.4)$ & $217(25.5)$ & $<0.001$ \\
\hline Maintenance dialysis (\%) & $7(1.5)$ & $3(0.6)$ & $3(0.4)$ & $2(0.2)$ & 0.008 \\
\hline Coronary artery disease (\%) & $8(1.7)$ & $5(1.1)$ & $18(2.5)$ & $31(3.6)$ & 0.004 \\
\hline Cerebrovascular disease (\%) & $3(0.6)$ & $11(2.4)$ & $16(2.2)$ & $23(2.7)$ & 0.020 \\
\hline
\end{tabular}

COPD, chronic obstructive pulmonary disease. 

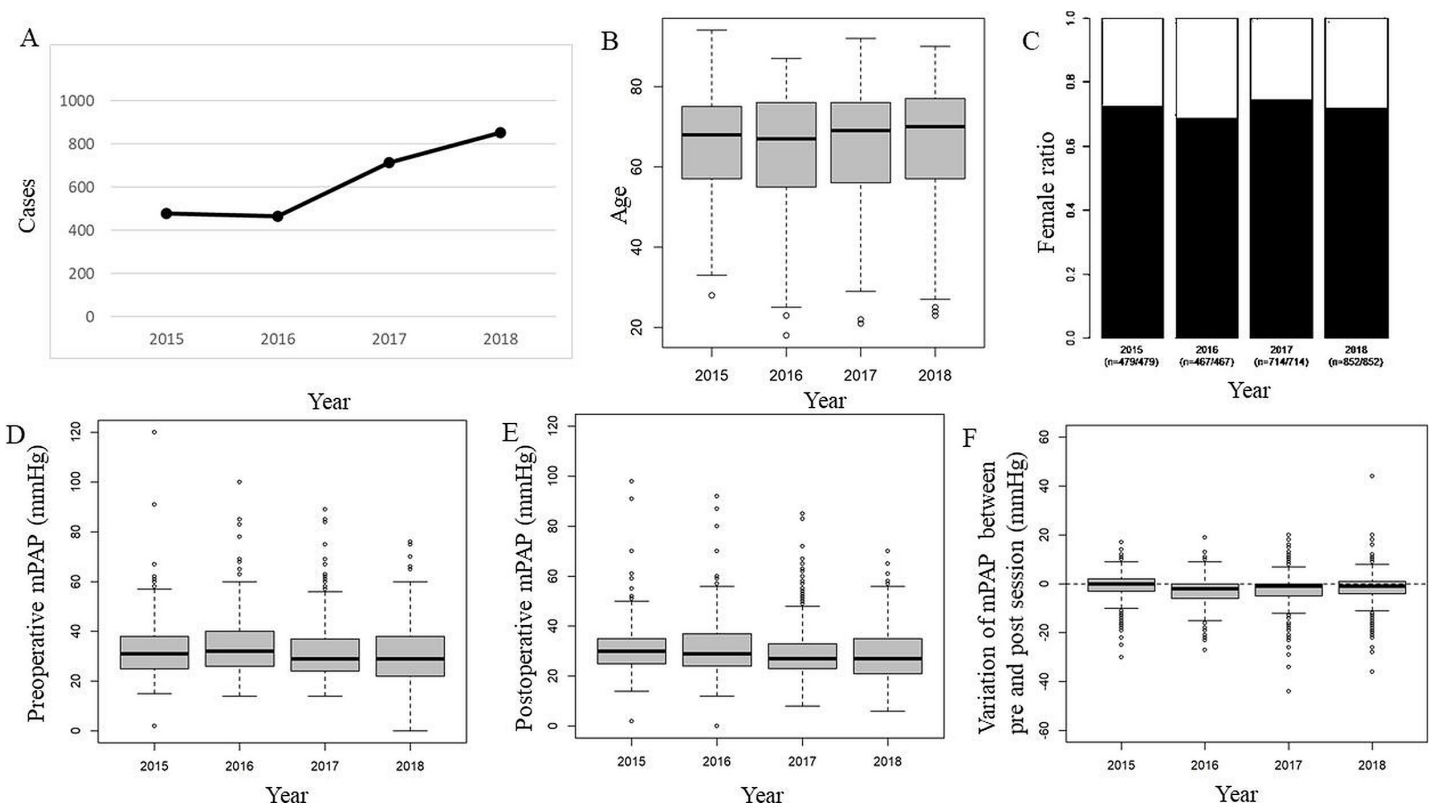

Figure 1 Time-trend analysis (2015-2018) of balloon pulmonary angioplasty (BPA) sessions for patients with chronic thromboembolic pulmonary hypertension. Data from the Japanese Structural Heart disease registry. A: Trend for the number of BPA sessions. B: Trend for patients' age. C: Trend for male to female ratio. D and E: Trend for preoperative and postoperative mPAP. F: Variation in mPAP between pre and post BPA sessions. mPAP, mean pulmonary artery pressure.

\section{Procedural results}

The success rate of the BPA sessions was $>98 \%$ (2015: 98.7\%; 2016: $98.1 \%$; 2017: 99.2\%; 2018: $99.5 \%$ ), and there was a trend towards an increased success rate ( $\mathrm{p}=0.044)$ over the study period. The rate of emergent BPA was low $(0.6 \%-1.5 \%)$, with a similar rate across all the registered years (2015: 1.5\%; 2016: 1.5\%; 2017: 0.6\%;
2018: $1.2 \% ; \mathrm{p}=0.43)$. The procedural results are shown in table 2.

The preoperative mean pulmonary artery pressure (mPAP) decreased during the study period (2015: 32 $\pm 11 \mathrm{~mm} \mathrm{Hg}$; 2016: 34 $\pm 11 \mathrm{~mm} \mathrm{Hg}$; 2017: 32 $\pm 11 \mathrm{~mm} \mathrm{Hg}$; 2018: $31 \pm 11 \mathrm{~mm} \mathrm{Hg} ; \mathrm{p}<0.001$; figure 1D). The postoperative mPAP exhibited a similar trend (2015: $31 \pm 9 \mathrm{~mm}$

\begin{tabular}{|c|c|c|c|c|c|}
\hline Year & 2015 & 2016 & 2017 & 2018 & $P$ for trend \\
\hline Number of institutions & 30 & 42 & 48 & 50 & \\
\hline Sessions & 479 & 467 & 714 & 852 & \\
\hline Emergent BPA (\%) & $7(1.5)$ & $7(1.5)$ & $4(0.6)$ & $10(1.2)$ & 0.43 \\
\hline Preoperative mPAP* $(\mathrm{mm} \mathrm{Hg})$ & $32 \pm 11$ & $34 \pm 12$ & $32 \pm 11$ & $31 \pm 11$ & 0.001 \\
\hline Median & $31(25-38)$ & $32(26-40)$ & $29(24-37)$ & $29(22-38)$ & \\
\hline Postoperative mPAP† (mm Hg ) & $31 \pm 9$ & $31 \pm 10$ & $29 \pm 10$ & $29 \pm 10$ & $<0.001$ \\
\hline Median & $30(25-35)$ & $29(24-37)$ & $27(23-33)$ & $27(21-35)$ & \\
\hline Operation time (min) & No data & No data & $183 \pm 57$ & $170 \pm 62$ & 0.005 \\
\hline Median & & & $182(141-222)$ & $162(116-215)$ & \\
\hline Fluoroscopic timeł (min) & $74 \pm 33$ & $88 \pm 52$ & $86 \pm 61$ & $80 \pm 48$ & 0.98 \\
\hline Median & $72(49-90)$ & $78(51-115)$ & $70(48-103)$ & $69(51-92)$ & \\
\hline Amount of contrast medium $\S(\mathrm{mL})$ & $173 \pm 70$ & $167 \pm 71$ & $166 \pm 69$ & $161 \pm 78$ & 0.007 \\
\hline Median & $170(120-215)$ & $165(115-210)$ & $160(120-200)$ & $150(110-190)$ & \\
\hline Successful BPA (\%) & $473(98.7)$ & $458(98.1)$ & 708 (99.2) & $848(99.5)$ & 0.044 \\
\hline $95 \% \mathrm{Cl}(\%)$ & $97.3-99.5$ & $96.4-99.1$ & $98.2-99.7$ & $98.8-99.9$ & \\
\hline
\end{tabular}

${ }^{*}$ The rate of missing data: $2015,0.2 \% ; 2016,0.4 \% ; 2017,0.6 \% ; 2018,9.2 \%$.

†2015, 0.4\%; 2016, 0.6\%; 2017, 0.6\%; 2018, 9.3\%.

$\ddagger 2015,49.9 \%$; 2016, 10.5\%; 2017, 24.8\%; 2018, 20.7\%.

$\S 2015,0.6 \%$; 2016, 0.0\%; 2017, 0.0\%; 2018, 0.0\%.

BPA, balloon pulmonary angioplasty; mPAP, mean pulmonary artery pressure. 
Table 3 Summary of complications of balloon pulmonary angioplasty for patients with chronic thromboembolic pulmonary hypertension and comparison between high-volume (performing more than 50 BPA sessions) and general institutions performing BPA sessions

\begin{tabular}{|c|c|c|c|c|c|}
\hline Year & 2015 & 2016 & 2017 & 2018 & Total \\
\hline Number of institutions & 30 & 42 & 48 & 50 & 120 \\
\hline Sessions & 479 & 467 & 714 & 852 & 2512 \\
\hline Complication (\%) * & $10(2.1)$ & $30(6.4)$ & $45(6.3)$ & $49(5.8)$ & $134(5.3)$ \\
\hline $95 \% \mathrm{Cl}(\%)$ & $1.0-3.8$ & $4.4-9.0$ & $4.6-8.3$ & $4.3-7.5$ & \\
\hline In-hospital death (\%) & $1(0.2)$ & $2(0.4)$ & $1(0.1)$ & $0(0.0)$ & $4(0.2)$ \\
\hline Required blood transfusion (\%) & $3(0.6)$ & $2(0.4)$ & $2(0.3)$ & $2(0.2)$ & $9(0.4)$ \\
\hline Emergent surgical treatment (\%) & $1(0.2)$ & $0(0.0)$ & $0(0.0)$ & $0(0.0)$ & $1(0.0)$ \\
\hline Distal embolism (\%) & $1(0.2)$ & $0(0.0)$ & $0(0.0)$ & $0(0.0)$ & $1(0.0)$ \\
\hline Vessel rupture (\%) & $5(1.0)$ & $6(1.3)$ & $6(0.8)$ & $6(0.7)$ & $23(0.9)$ \\
\hline Acute obstruction (\%) & $1(0.2)$ & $0(0.0)$ & $0(0.0)$ & $0(0.0)$ & $1(0.0)$ \\
\hline Contrast-induced nephropathy (\%) & $1(0.2)$ & $0(0.0)$ & $1(0.1)$ & $0(0.0)$ & $2(0.1)$ \\
\hline Others (\%) & $7(1.5)$ & $23(4.9)$ & $37(5.2)$ & $42(4.9)$ & $109(4.3)$ \\
\hline 'Bloody sputum' or 'lung bleeding' & $3(0.6)$ & $20(4.2)$ & $29(4.1)$ & $25(2.9)$ & $77(3.0)$ \\
\hline Cardiac shock required mechanical support & No data & No data & No data & $0(0.0)$ & $0(0.0)$ \\
\hline \multicolumn{6}{|l|}{ Number of facilities } \\
\hline High volume (\%) & $2(6.7)$ & $2(4.8)$ & $3(6.3)$ & $6(12.0)$ & \\
\hline General (\%) & $28(93.3)$ & $40(95.2)$ & $45(93.7)$ & $44(88.0)$ & \\
\hline \multicolumn{6}{|l|}{ Number of BPA session } \\
\hline High volume (\%) & $229(47.8)$ & $151(32.3)$ & 249 (34.9) & $451(52.9)$ & \\
\hline General (\%) & $250(52.2)$ & $316(67.7)$ & $465(65.1)$ & $401(47.1)$ & \\
\hline \multicolumn{6}{|l|}{ Incidence of complications (\%) } \\
\hline High volume & 0.9 & 6.0 & 10.4 & 6.7 & 6.2 \\
\hline General & 3.2 & 6.6 & 4.1 & 4.7 & 4.7 \\
\hline$P$ value & 0.14 & 0.94 & 0.002 & 0.29 & 0.11 \\
\hline
\end{tabular}

${ }^{*} p=0.017$

BPA, balloon pulmonary angioplasty.

Hg; 2016: 31 $\pm 10 \mathrm{~mm} \mathrm{Hg;} \mathrm{2017:} \mathrm{29 \pm 10} \mathrm{mm} \mathrm{Hg;} \mathrm{2018:}$ $29 \pm 10 \mathrm{~mm} \mathrm{Hg} ; \mathrm{p}<0.001$; figure $1 \mathrm{E})$. In each session, the average variation of mPAP between the pre and post session was $-2.0 \pm 5.2 \mathrm{~mm} \mathrm{Hg}(\mathrm{p}<0.001$; figure $1 \mathrm{~F})$, excluding three sessions that did not register both premPAP and post-mPAP. The average fluoroscopic time was not different (2015: 73 $\pm 33 \mathrm{~min}$; 2016: $88 \pm 52 \mathrm{~min}$; 2017 : $86 \pm 61 \mathrm{~min}$; 2018: $80 \pm 48 \mathrm{~min}$; $\mathrm{p}=0.98$ ), while the amount of contrast medium significantly decreased every year (2015: $173 \pm 70 \mathrm{~mL} ; 2016: 167 \pm 71 \mathrm{~mL} ; 2017: 166 \pm 69 \mathrm{~mL}$; 2018: $161 \pm 78 \mathrm{~mL} ; \mathrm{p}<0.007)$.

\section{Complication rates}

The summary of complication rates is shown in table 3 .

The occurrence rate of complications was roughly $6 \%$ every year (2015: 2.1\%; 2016: 6.4\%; 2017: 6.3\%; 2018: $5.8 \% ; \mathrm{p}=0.017)$. In-hospital death occurred in four sessions (2015: one session; 2016: two sessions; 2017: one session; 2018: none; $0.2 \%$ overall). The 'Others' complications were divided into three categories. The first was 'perforation induced by guidewire' (2015: one session; 2016: two sessions; 2017: five sessions; 2018: nine sessions). Among them, some specified the occurrence of bloody sputum (2015: no session; 2016: two sessions; 2017: two sessions; 2018: no session). The second was 'injury (dissection) of the pulmonary artery induced by the guiding catheter or balloon inflation' (2015: no session; 2016: one session; 2017: no session; 2018: five sessions). Only one session (2016) specified the occurrence of bloody sputum. The third was 'bloody sputum' or 'lung bleeding' (2015: 3 sessions; 2016: 17 sessions; 2017: 26 sessions; 2018: 25 sessions).

\section{Analysis of the number of complications by the volume of the institution}

The number of sessions with complication in the highvolume group was two in 2015 (6.7\%), two in 2016 $(4.8 \%)$, three in $2017(6.3 \%)$ and six in $2018(12.0 \%)$. Regarding the number of BPA sessions, the high-volume group accounted for 229 sessions in 2015 (47.8\%), 151 sessions in 2016 (32.3\%), 249 sessions in 2017 (34.9\%) and 451 sessions in 2018 (52.9\%). The total incidence of 
complications was not statistically different between the two groups (high-volume group: $6.2 \%$ vs general group: $4.7 \%$; $\mathrm{p}=0.11$; table 3 ).

\section{DISCUSSION}

Our results demonstrate the current status and trends in BPA in Japan. During the study period, the number of patients who underwent BPA increased, and their mPAP showed a downward trend. The rate of in-hospital deaths was $0.2 \%$ and that of complications was roughly $6 \%$. Although the real-world data of riociguat have been previously reported, ${ }^{22}$ this is the first report demonstrating real-world trends affecting BPA in numerous cases using a nationwide registry.

\section{Patient characteristics}

As for the patient characteristics, a report from the Japan Intractable Diseases Information Center indicated that the average age of the patients was $66 \pm 13$ years, and the number of female patients was approximately three times the number of male patients in 2013 (http://www. nanbyou.or.jp/entry/192). Our results showed a similar tendency regarding the current situation of CTEPH in Japan. The number of cases tended to increase every year in our registry. The annual report in 2018 from the Japanese Registry Of All cardiac and vascular Diseases (http:/ / www.j-circ.or.jp/jittai_chosa/jittai_chosa2018web.pdf), which is a portal site for the Japanese Circulation Society, showed the same tendency; however, a higher number of BPA sessions were registered in this registry (2015: 1752 sessions; 2016: 1910 sessions; 2017: 2294 sessions; 2018: 2470 sessions) than those in our registry. This indicates that our registry could not cover all BPA sessions.

\section{Preoperative and postoperative mPAP}

Preoperative mPAP decreased during the evaluated years $(p<0.001)$ in our registry. We have two speculations regarding this result. First, with the expansion of the indications for BPA, the number of BPA procedures increased. Second, an increasing number of patients were administered riociguat before BPA. Riociguat, which is a soluble guanylate cyclase agonist, improves both pulmonary vascular resistance and exercise tolerance ${ }^{23}$ and has been approved in Japan for patients with CTEPH since January 2014. The current therapeutic algorithm for CTEPH strongly recommends riociguat administration for inoperable CTEPH cases. ${ }^{24}$ An increase in the number of registered cases and a decrease in mPAP over time during the evaluated years were considered to indicate the degree of awareness regarding CTEPH. Postoperative mPAP, which is measured when a BPA session is finished, also showed a decreasing trend $(\mathrm{p}<0.001)$; however, the improvement of mPAP in one session was quite small (1-2 mm Hg) compared with that in previous reports of mPAP in the follow-up period. We have three speculations to explain this. First, the preoperative mPAP in our analysis was low. Second, the time from balloon dilatation to the measurement of postoperative mPAP could have been short. After balloon dilatation, the pulmonary arteries self-expand over time because the blood flow to the distal side of the stenosis is increased, which induces further improvement in mPAP. ${ }^{25}$ There is a possibility that self-expansion of the treated vessels cannot fully occur at the time of the postoperative mPAP measurement. Third, there is a possibility of micro-lung injury induced by wire injury to microvessels. Micro-lung injury might elevate pulmonary vascular resistance without causing symptoms such as haemoptysis and deterioration of arterial oxygen saturation.

\section{In-hospital death rate}

In this study, the in-hospital death rate ranged from $0.1 \%$ to $0.2 \%$. The in-hospital death rate in the initial report of BPA was $5.6 \% .{ }^{26}$ It is thought that this is largely due to the development of the procedural technique. In a previous multicentre registry that represented the early stage of BPA in Japan, eight patients (2.6\%) died within 30 days of BPA and four died during the follow-up period. ${ }^{2}$ There may be three reasons for the low mortality rate in our results. One is the difference in calculation method. Our results were calculated by death/session numbers, while previous reports were calculated by death/patient number. Second is the difference in the patients' condition. This preceding multicentre registry comprised only BPA expert centres, and it is possible that patients with poor haemodynamics and severe comorbidities could have been targeted. Third is the relationship between haemodynamic improvement effects and the complications in BPA. In the above report, mPAP improved from $43.2 \pm 11.0 \mathrm{~mm} \mathrm{Hg}$ to $22.5 \pm 5.4 \mathrm{~mm} \mathrm{Hg}$, while the incidence of lung injury was $17.8 \%$. Two European registries ${ }^{11} 27$ had less improvement in mPAP (from $40 \pm 12 \mathrm{~mm} \mathrm{Hg}$ to $33 \pm 11 \mathrm{~mm} \mathrm{Hg}$ and from $43.9 \pm 9.5 \mathrm{~mm} \mathrm{Hg}$ to $31.6 \pm 9.0 \mathrm{~mm}$ $\mathrm{Hg}$, respectively) and a lower incidence of lung injury (9.4\% and $9.1 \%$, respectively) than those in the Japanese registry. This may be a procedural issue in BPA, where if more peripheral lesions are treated to further improve haemodynamics, the possibility of lung injury due to wire perforation increases. Our registry is mainly composed of non-expert centres (88.0\%-95.2\% of all facilities), and it is thought that this is the result of postponing the treatment of peripheral lesions, emphasising safety. This tendency was consistent with our results of examining complication rates at the expert institution and general institution. Besides, this result could indicate that the complication rate seems to be acceptable even when BPA is not performed in a high-volume expert centre.

\section{Limitations}

This study has some limitations. First, it was difficult to analyse clinical data such as patient characteristics, namely, rate of administration of pulmonary hypertension-specific drugs and rate of residual pulmonary hypertension after pulmonary endarterectomy and haemodynamic data because this database was aggregated by BPA session not by patient. Second, BPA-specific 
complications, such as pulmonary haemorrhage and lung injury, may have been under-reported. Because there is no specific choosing option for these adverse effects of BPA in the registry registration forms, the registrants had to fill these in other fields themselves. Further investigation is needed to evaluate BPA-specific complications and their prognostic factors. Third, preoperative mPAP in previous reports is usually $>40 \mathrm{~mm} \mathrm{Hg}$, which is higher than that in our report. This is because our preoperative mPAP data represent mPAP measured before each BPA session and not the initial data from each patient. Fourth, the definition of emergent BPA and in-hospital deaths was not clear. We dealt with this by setting a wide range of definitions. Fifth, data regarding long-term follow-up were not available. This registry is registered per BPA session and not per case. Further investigation is needed to evaluate the real-world outcomes, effectiveness and safety of BPA. Sixth, although the database managers underwent training on an annual basis during our scientific sessions, the data were not internally validated by a third party. There are few nationwide databases on BPA in Japan. Finally, because the number of variables was limited in the J-SHD registry, unknown and unevaluated confounders, especially other haemodynamic details, medications and procedural details, including treated lung segments, could affect the outcomes.

\section{CONCLUSIONS}

The number of BPA sessions for patients with CTEPH increased during the study period, with an acceptable in-hospital complication rate. Our study demonstrates that with some limitations, BPA is a feasible and safe therapeutic method treating patients with CTEPH. Further studies improvising on the limitations of our study are required to ascertain our results.

\section{Author affiliations}

${ }^{1}$ Department of Cardiovascular Medicine, Graduate School of Medicine, The University of Tokyo, Bunkyo-ku, Tokyo, Japan

${ }^{2}$ Department of Diabetes Care Medicine, Osaka University Graduate School of Medicine, Suita, Osaka, Japan

${ }^{3}$ Department of Therapeutic Strategy for Heart Failure, Graduate School of

Medicine, The University of Tokyo, Bunkyo-ku, Tokyo, Japan

${ }^{4}$ Department of Cardiology, Keio University School of Medicine, Shinjuku-ku, Tokyo, Japan

${ }^{5}$ Department of Cardiology, Fujita Health University Bantane Hospital, Nagoya, Aichi, Japan

${ }^{6}$ Department of Cardiology, Showa University School of Medicine, Shinagawa-ku, Tokyo, Japan

${ }^{7}$ Department of Cardiology, Aichi Medical University, Nagakute, Aichi, Japan

${ }^{8}$ Cardiovascular Medicine, Tokai University Hospital, Isehara, Kanagawa, Japan

Acknowledgements The authors appreciate the contribution of all the investigators, clinical coordinators and institutions involved in the J-SHD registry.

Collaborators The contribution of all the investigators, clinical coordinators, and institutions involved in the J-SHD and the J-PCl registry (full list of institutions provided in http://www.cvit.jp/registry/research_longterm_prognosis.html).

Contributors SM, MT, AK, SK, MH, JA and SK contributed the conception and design or analysis and interpretation of data, $\mathrm{HI}, \mathrm{TS}, \mathrm{TA}, \mathrm{YI}$ and IK contributed drafting of the manuscript or revising it critically for important intellectual content, and SK contributed final approval of the manuscript submitted.
Funding This study was funded by the Grant-in-Aid from Scientific Research from the Japan Agency for Medical Research and Development (grant no. 17ek0210097h000) and the Japan Society for the Promotion of Science (grant no. 20H03915).

Competing interests None declared.

Patient consent for publication Not required.

Ethics approval This study protocol was approved by the relevant committee and the Board of Directors of the CVIT. The data analysis was performed in accordance with the Declaration of Helsinki and was approved by the ethics committee of the Clinical Research Promotion Network Japan (approval number CRPNJ_SOP-4).

Provenance and peer review Not commissioned; externally peer reviewed.

Data availability statement Data are available upon reasonable request. The data elements were collected from the Japanese Structural Heart Disease (J-SHD) registry, which was established in 2012 and is a prospective Japanese nationwide multicentre registry founded by the Japanese Association of Cardiovascular Intervention and Therapeutics (CVIT) designed to collect clinical variables and short-term outcome data on patients with SHD. The mission of CVIT is to further our understanding of cardiovascular intervention and establish its procedural safety. Today, the database has grown to become one of the largest healthcare procedural database with more than $20000 \mathrm{SHD}$ intervention cases registered annually (excluding transcatheter aortic valve replacement, which is registered onto J-TVT registry) from approximately 200 institutions in Japan representing more than $90 \%$ of all SHD intervention-performing hospitals in the nation. Importantly, case registrations in the J-SHD registry are essential for coronary interventionalist and educating hospital certification. This study protocol was approved by the institutional review board (http://www.cvit.jp/files/registry/central_irb.pdf). The BPA data, which are one of the components of the J-SHD, were collected from 2015. The CVIT registry subcommittee designed a software for a web-based data collection system through the National Clinical Database. Data managers in the participating hospitals submit data through this system annually. Because registration in the J-SHD database is mandatory for board certification and renewal applications, data completeness is high, although participation in the J-SHD is voluntary. This study protocol was approved by the relevant committee and the Board of Directors of the CVIT.

Open access This is an open access article distributed in accordance with the Creative Commons Attribution Non Commercial (CC BY-NC 4.0) license, which permits others to distribute, remix, adapt, build upon this work non-commercially, and license their derivative works on different terms, provided the original work is properly cited, appropriate credit is given, any changes made indicated, and the use is non-commercial. See: http://creativecommons.org/licenses/by-nc/4.0/.

ORCID iDs

Shun Minatsuki http://orcid.org/0000-0002-9543-5503

Hideki Ishii http://orcid.org/0000-0003-4348-0123

\section{REFERENCES}

1 Mizoguchi H, Ogawa A, Munemasa M, et al. Refined balloon pulmonary angioplasty for inoperable patients with chronic thromboembolic pulmonary hypertension. Circ Cardiovasc Interv 2012;5:748-55

2 Ogawa A, Satoh T, Fukuda T, et al. Balloon pulmonary angioplasty for chronic thromboembolic pulmonary hypertension: results of a multicenter registry. Circ Cardiovasc Qual Outcomes 2017;10.

3 Sugimura K, Fukumoto $\mathrm{Y}$, Satoh K, et al. Percutaneous transluminal pulmonary angioplasty markedly improves pulmonary hemodynamics and long-term prognosis in patients with chronic thromboembolic pulmonary hypertension. Circ J 2012;76:485-8.

4 Andreassen AK, Ragnarsson A, Gude E, et al. Balloon pulmonary angioplasty in patients with inoperable chronic thromboembolic pulmonary hypertension. Heart 2013;99:1415-20.

5 Kataoka M, Inami T, Hayashida K, et al. Percutaneous transluminal pulmonary angioplasty for the treatment of chronic thromboembolic pulmonary hypertension. Circ Cardiovasc Interv 2012;5:756-62.

6 Bouvaist $\mathrm{H}$, Thony $\mathrm{F}$, Jondot $\mathrm{M}$, et al. Balloon pulmonary angioplasty in a patient with chronic thromboembolic pulmonary hypertension. Eur Respir Rev 2014;23:393-5.

7 Kozu K, Sugimura K, Ito M, et al. Current status of long-term prognosis among all subtypes of pulmonary hypertension in Japan. Int J Cardiol 2020;300:228-35.

8 Minatsuki S, Kodera S, Kiyosue A, et al. Balloon pulmonary angioplasty improves quality of life in Japanese patients with chronic thromboembolic pulmonary hypertension. J Cardiol 2020;76:205-10. 
9 Galiè N, Humbert M, Vachiery J-L, et al. 2015 ESC/ERS guidelines for the diagnosis and treatment of pulmonary hypertension: the joint Task force for the diagnosis and treatment of pulmonary hypertension of the European Society of cardiology (ESC) and the European respiratory Society (ERS): endorsed by: association for European paediatric and congenital cardiology (AEPC), International Society for heart and lung transplantation (ISHLT). Eur Heart J 2016;37:67-119. doi:10.1093/eurheartj/ehv317

10 Roik M, Wretowski D, Rowiński O, et al. Refined balloon pulmonary angioplasty in inoperable chronic thromboembolic pulmonary hypertension--a multi-modality approach to the treated lesion. Int J Cardiol 2014;177:e139-41.

11 Brenot $\mathrm{P}$, Jaïs $\mathrm{X}$, Taniguchi $\mathrm{Y}$, et al. French experience of balloon pulmonary angioplasty for chronic thromboembolic pulmonary hypertension. Eur Respir J 2019;53. doi:10.1183/13993003.020952018. [Epub ahead of print: 18 May 2019].

12 Pepke-Zaba J, Delcroix M, Lang I, et al. Chronic thromboembolic pulmonary hypertension (CTEPH): results from an international prospective registry. Circulation 2011;124:1973-81.

13 Peacock A, Simonneau G, Rubin L. Controversies, uncertainties and future research on the treatment of chronic thromboembolic pulmonary hypertension. Proc Am Thorac Soc 2006;3:608-14.

14 Mayer E, Jenkins D, Lindner J, et al. Surgical management and outcome of patients with chronic thromboembolic pulmonary hypertension: results from an international prospective registry. $J$ Thorac Cardiovasc Surg 2011;141:702-10.

15 Committee for Scientific Affairs, Sakata R, Fujii Y, et al. Thoracic and cardiovascular surgery in Japan during 2009: annual report by the Japanese association for thoracic surgery. Gen Thorac Cardiovasc Surg 2011;59:636-67.

16 Vestbo J, Hurd SS, Agustí AG, et al. Global strategy for the diagnosis, management, and prevention of chronic obstructive pulmonary disease: gold executive summary. Am J Respir Crit Care Med 2013;187:347-65.

17 Shimamoto K, Ando K, Fujita T, et al. The Japanese Society of Hypertension guidelines for the management of hypertension (JSH 2014). Hypertens Res 2014;37:253-390.
18 Haneda M, Noda M, Origasa $\mathrm{H}$, et al. Japanese clinical practice guideline for diabetes 2016. Diabetol Int 2018;9:1-45.

19 Teramoto T, Sasaki J, Ishibashi S, et al. Executive summary of the Japan Atherosclerosis Society (JAS) guidelines for the diagnosis and prevention of atherosclerotic cardiovascular diseases in Japan -2012 version. J Atheroscler Thromb 2013;20:517-23.

20 Sawano M, Yamaji K, Kohsaka S, et al. Contemporary use and trends in percutaneous coronary intervention in Japan: an outline of the J-PCI registry. Cardiovasc Interv Ther 2020;35:218-26.

21 Mohammed NMA, Mahfouz A, Achkar K, et al. Contrast-Induced nephropathy. Heart Views 2013;14:106-16.

22 Tanabe N, Ogo T, Hatano M, et al. Safety and effectiveness of riociguat for chronic thromboembolic pulmonary hypertension in real-world clinical practice: interim data from post-marketing surveillance in Japan. Pulm Circ 2020;10:2045894020938986.

23 Ghofrani H-A, D'Armini AM, Grimminger F, et al. Riociguat for the treatment of chronic thromboembolic pulmonary hypertension. $N$ Engl J Med 2013;369:319-29.

24 Wilkens $\mathrm{H}$, Konstantinides S, Lang IM, et al. Chronic thromboembolic pulmonary hypertension (CTEPH): updated recommendations from the Cologne Consensus Conference 2018. Int J Cardiol 2018;272S: 272s:69-78.

25 Nagayoshi S, Ogawa A, Matsubara H. Spontaneous enlargement of pulmonary artery after successful balloon pulmonary angioplasty in a patient with chronic thromboembolic pulmonary hypertension. Eurolntervention 2016;12:e1435.

26 Feinstein JA, Goldhaber SZ, Lock JE, et al. Balloon pulmonary angioplasty for treatment of chronic thromboembolic pulmonary hypertension. Circulation 2001;103:10-13.

27 Olsson KM, Wiedenroth CB, Kamp J-C, et al. Balloon pulmonary angioplasty for inoperable patients with chronic thromboembolic pulmonary hypertension: the initial German experience. Eur Respir J 2017;49. doi:10.1183/13993003.02409-2016. [Epub ahead of print: 08 Jun 2017]. 